\title{
ANÁLISE DE DIABETES MELLITUS E HIPERTENSÃO ARTERIAL SISTÊMICA EM CRIANÇAS E ADOLESCENTES: ESTUDO EPIDEMIOLÓGICO
}

\author{
ANALYSIS OF DIABETES MELLITUS AND SYSTEMIC ARTERIAL \\ HYPERTENSION IN CHILDREN AND ADOLESCENTS: AN \\ EPIDEMIOLOGICAL STUDY
}

\author{
Nertan Ribeiro Batista ${ }^{1}$ \\ Ozeny de Sousa Moura² \\ Francisco Samuel Torquato Lima ${ }^{3}$ \\ Pollianna Marys de Souza e Silva ${ }^{4}$ \\ Elisangela Vilar de Assis ${ }^{5}$
}

Resumo: O objetivo desta pesquisa é avaliar a prevalência de crianças e adolescentes com diabetes mellitus (DM) e hipertensão arterial sistêmica (HAS). Assim, essa pesquisa é um estudo ecológico, retrospectivo e epidemiológico, realizados com dados do Departamento de Informática do Sistema Único de Saúde (DATASUS) referentes às crianças e adolescentes do estado da Paraíba com DM e HAS, além da associação entre estas, no período de abr/2011 a abr/2013. Foram considerados variáveis do estilo de vida: sedentarismo, sobrepeso e tabagismo. 0 programa SPSS (versão 25) foi a ferramenta utilizada para analisar os dados da pesquisa. Foi obtida maior porcentagem de crianças e adolescentes do sexo masculino e mais novos com DM e HAS quando comparado com o sexo feminino, porém sem associação estatisticamente significativa. $\mathrm{Na}$ análise entre sexo e idade, conforme estilo de vida, também não houve associação estatisticamente significativa em ambos os casos. Entre as crianças e adolescentes hipertensos, observou-se que existem menos meninas tabagistas na faixa etária de 15 a 19 anos. Entre os hipertensos e diabéticos, nessa mesma faixa etária houve predomínio de tabagismo nos meninos. Dessa forma, a associação com estilo de vida de adolescentes e

\footnotetext{
1 Universidade Federal de Campina Grande, Cajazeiras, Paraíba, Brasil. E-mail: nertan123@gmail.com.

2 Universidade Federal de Campina Grande, Cajazeiras, Paraíba, Brasil. E-mail: ozenysousa@gmail.com.

3 Universidade Federal de Campina Grande, Cajazeiras, Paraíba, Brasil. E-mail: samueltorquato0@gmail.com.

${ }^{4}$ Secretaria Estadual de Saúde, João Pessoa, Paraíba, Brasil. E-mail: pollianna_marys@hotmail.com.

5 Universidade Federal de Campina Grande, Cajazeiras, Paraíba, Brasil. E-mail: ely.vilar@hotmail.com.
} 
presença de doenças cardiovasculares (DCV) não foi estatisticamente significativa em nosso estudo, porém a HAS isolada e o conjunto HAS e DM foram mais prevalentes nos meninos. A falta de dados mais atuais no DATASUS dificultou a percepção do panorama desejado e representa fator impeditivo em relação a políticas públicas voltadas para a população estudada.

Palavras-chave: Análise de Situação de Saúde; Criança e Adolescente; Hipertensão Arterial Sistêmica; Diabetes Mellitus.

Abstract: The aim of this research is to evaluate the prevalence of children and adolescents with diabetes mellitus (DM) and systemic arterial hypertension (SAH). Thus, this research is an ecological, retrospective and epidemiological study, conducted with data from the Department of Informatics of the Unified Health System (DATASUS) referring to children and adolescents in the State of Paraiba with DM and $S A H$, in addition to the association between them, in the period from Apr/2011 to Apr/2013. Lifestyle variables were considered: sedentary lifestyle, overweight and smoking. The SPSS program (version 25) was the tool used to analyze the research data. A higher percentage of male and younger children and adolescents with DM and SAH was obtained when compared to females, but without statistically significant association. In the analysis between gender and age, according to lifestyle, there was also no statistically significant association in both cases. Among hypertensive children and adolescents, it was observed that there are fewer smoking girls in the age group from 15 to 19. Among hypertensive and diabetic patients, in this same age group there was a predominance of smoking in boys. Thus, the association with adolescent lifestyle and the presence of cardiovascular diseases (CVD) was not statistically significant in our study, but isolated SAH and SAH and DM were more prevalent in boys. The lack of more current data in DATASUS made it difficult to perceive the desired panorama and represents an preventing factor in relation to public policies aimed at the population studied.

Keywords: Child and Teenager; Health Situation Analysis; Systemic Arterial Hypertension; Diabetes Mellitus. 


\section{INTRODUÇÃO}

Desde o período de 1960 as DCV representam uma das principais causas de morte no Brasil e, atualmente, respondem por dois terços de todas as mortes. Diversos motivos contribuem para o desenvolvimento dessas doenças, tais como diabetes mellitus (DM), sobrepeso, hipertensão arterial sistêmica (HAS) e dislipidemia e embora sejam mais associadas aos adultos e idosos, também são identificadas em pessoas mais jovens (FONTES; NEVES; CÂNDIDO, 2018).

As DCV são responsáveis por incapacidade e mortalidade prematuras (BRASIL, 2011), constituindo um grupo de enfermidades que afetam o coração e os vasos sanguíneos, como, por exemplo, a doença isquêmica do coração e a doença cerebrovascular. A sua etiologia é multifatorial, estando envolvidos fatores de risco comportamentais (dieta não saudável, inatividade física, tabagismo e uso de álcool), metabólicos (obesidade e pressão, colesterol e glicemia altos), além de fatores genéticos, sociais e psicológicos, idade e sexo (BRITO et al., 2016).

A quantidade de mortes causadas por um estilo de vida sedentário pode chegar a quase cinco milhões de indivíduos em todo o mundo, sendo que, em adolescentes, a preponderância do sedentarismo também é alta. O Estudo dos Riscos Cardiovasculares em Adolescentes (ERICA) demonstrou que existe uma frequência de $54 \%$ de sedentarismo, com predominância em jovens do sexo feminino (SCHERR et al., 2019).

Um dos principais contribuintes para o desencadeamento das DCV é a aterosclerose. Vale destacar que esse processo aterosclerótico é iniciado ainda quando criança e seu risco é proporcional ao número associado de fatores de risco. Portanto, a alimentação e a presença de exercícios físicos na infância e na adolescência têm potencial na vida adulta, visto que há evidências de que a aterosclerose começa na infância e progride lentamente até a idade adulta (SCHERR et al., 2019). 
A adolescência é um período de transição, compreendido entre os 10 e 19 anos é e marcado por mudanças em diferentes campos e níveis da vida do indivíduo, as quais requerem adaptações, fundamentais no processo dinâmico que é a saúde, de modo que essa fase pode apresentar repercussões na vida futura (SENNA; DESSEN, 2015). Assim, é importante analisar a ocorrência dos fatores de risco nesse estágio do desenvolvimento, o que pode estar relacionado às condições socioeconômicas e ao sexo do adolescente (FARIAS JÚNIOR et al., 2011), bem como ao estilo de vida adotado por influência de grupos sociais e pela impulsividade de experimentar o novo, podendo persistir nas fases subsequentes da vida (OLIVEIRA et al., 2019).

Estudos epidemiológicos são usados como ferramentas de fortalecimento e enfrentamento ao processo saúde/doença, pois auxiliam os gestores e facilitam o processo de tomada de decisão, através da construção de perfis epidemiológicos. A observação e identificação das condições de saúde de uma determinada região por meio desses perfis, obtidos a partir dos Sistemas de Informação em Saúde (SIS), são fatores importantes que possibilitam, além do direcionamento de estratégias e políticas públicas em saúde; o planejamento de toda rede de atenção (SILVA; CIRINO NETO; MIRANDA, 2020).

Silva e Autran (2019) relatam que os SIS são instrumentos fundamentais para definir e planejar questões de saúde, pois proporcionam acesso livre a conteúdos e informações que podem guiar intervenções e também avaliar o impacto dessas, através do fornecimento dos indicativos das causas e dos riscos (doenças/agravos) aos quais as pessoas estão expostas; contribuindo dessa forma para o reconhecimento epidemiológico de determinado espaço geográfico.

Dessa forma, Bittar et al. (2018) enfatizam a importância do uso dos SIS, para o aperfeiçoamento da assistência em saúde, através da elaboração contínua de pesquisas e estudos que auxiliarão os profissionais na tomada de decisão administrativa e também no cuidado imediato.

Devido aos fatores supracitados, nota-se a relevância desta pesquisa que tem como objetivo avaliar a frequência e porcentagem de crianças e adolescentes com diabetes mellitus (DM) e hipertensão arterial sistêmica (HAS) no Repositório Institucional DATASUS. 
O RI do Departamento de Informática do Sistema de Único de Saúde (SUS), - DATASUS, pertencente ao Ministério da Saúde (MS), é alimentado por uma série de SIS, cada qual encarregado pelo recolhimento das variadas áreas de amplitude do Sistema Único de Saúde (SUS). Este RI está responsável por regularizar, alicerçar e publicar as informações desenvolvidas em coletividade pelos três entes federativos, através do transporte eletrônico de dados gerando indicadores operacionais e/ou epidemiológicos (SILVA; AUTRAN 2019).

Tendo em vista que as Doenças Cardiovasculares Adquiridas (DCV) constituem a principal causa de morbidade e mortalidade no Brasil e no mundo e que o seu início pode ser atribuído na adolescência e no estilo de vida precário escolhido. Se faz necessário analisar esta questão sob a perspectiva de crianças e adolescentes, que podem ter uma qualidade de vida cada vez mais prejudicada e sofrerem com os sintomas desse problema por toda a vida, com possibilidade de ficarem impossibilitados de trabalhar devido às sequelas adquiridas. Ademais, é importante pontuar a alta demanda de recursos direcionados pelos governos para tratar as pessoas que são acometidas por essas doenças, impactando, assim, a sociedade como um todo.

Assim, o objetivo dessa pesquisa foi avaliar a prevalência de crianças e adolescentes com diabetes mellitus (DM) e hipertensão arterial sistêmica (HAS).

\section{MÉTODO}

Trata-se de um estudo ecológico, que permite a comparação de grupos ao invés de indivíduos, comumente empregados quando dados individuais não são disponíveis, com o intuito de gerar ou testar hipóteses etiológicas, explicar a ocorrência da doença, e avaliar a efetividade de intervenções na população (FRONTEIRAS, 2013).

Neste estudo foram utilizados dados primários do Departamento de Informação do Sistema Único de Saúde (DATASUS), referentes a Informações de Saúde (TABNET) no subitem Epidemiologia e Morbidade de crianças e adolescentes 
com HAS, DM e associação de HAS e DM, considerando variáveis relacionadas ao estilo de vida, como: sedentarismo, sobrepeso e tabagismo. Por tratar-se de um banco de dados de domínio público, não necessita avaliação por Comitê de Ética em Pesquisa.

Foram considerados os dados referentes ao estado da Paraíba no período de abril de 2011 a abril de 2013, período escolhido por constituir os dados mais recentes disponibilizados no sistema. As variáveis demográficas selecionadas foram sexo e faixa etária. A população estudada foi dividida em dois grupos: crianças e adolescentes com idade até 14 anos e adolescentes com idade entre 15 e 19 anos.

Os dados foram armazenados e analisados no Statistical Package for the Social Sciences SPSS (versão 25), através de estatística descritiva de frequência relativa e absoluta. Utilizou-se o teste inferencial de Qui-quadrado de Pearson, com significância estatística adotada de $p<0,05$.

\section{RESULTADOS E DISCUSSÃO}

Na tabela 1 é possível observar maior índice de crianças e adolescentes do sexo masculino hipertensos mais jovens, se comparados às crianças e adolescentes do sexo feminino. Porém, sem associação estatisticamente significativa.

Tabela 1 - Associação entre sexo e faixa etária de crianças e adolescentes com HAS no período de abril de 2011 a abril de 2013, Paraíba, Brasil.

\begin{tabular}{lccccc}
\hline \multirow{2}{*}{ Sexo } & \multicolumn{2}{c}{ Até 14} & \multicolumn{2}{c}{15 a 19} & \multirow{2}{*}{ Total } \\
\cline { 2 - 5 } & $\mathrm{f}$ & $\%$ & $\mathrm{f}$ & $\%$ & \\
\hline Masculino & 16 & $\mathbf{5 9 , 2 6}$ & 11 & 40,74 & 27 \\
Feminino & 15 & 41,67 & 21 & $\mathbf{5 8 , 3 3}$ & 36 \\
$\left(X^{2}\right)=$ significância & \multicolumn{3}{c}{$(1,91)=0,17$} \\
\hline
\end{tabular}

Fonte: Construção dos autores, 2021.

A HAS na adolescência tem se configurado como um relevante problema de saúde pública no Brasil. Em revisão sistemática com metanálise Gonçalves et al. (2016) constataram uma prevalência de $8 \%$ de indivíduos acometidos entre 
adolescentes escolares brasileiros, sendo esse valor superior no sexo masculino. Contudo, como em outros estudos, não houve diferença em relação à fase da adolescência e ao estágio de maturação sexual, dificultando análise comparativa mais precisa com os achados dessa pesquisa.

Costa et al. (2017) relatam que entre adolescentes com idades entre 15 e 19 anos a prevalência é maior de HAS nos homens, evidenciando como causa a diferença hormonal entre as pessoas do sexo feminino, pois essas possuem o estrógeno, que é um hormônio com potencial de controle do colesterol. Para Marte e Santos (2007), esse é um fator contributivo para os casos de HAS, haja vista que a elevada quantidade de lipídio pode interromper a produção de óxido nítrico, que é responsável pelo controle da vasodilatação e o controle da resistência vascular.

O paciente com dislipidemia possui elevada quantidade de radicais livres, que têm a capacidade de inativar algumas enzimas responsáveis pela produção e liberação de óxido nítrico (MARTE; SANTOS, 2007). Além disso, pesquisas anteriores evidenciaram que a pressão arterial é mais controlada nas mulheres, tal achado deveu-se a uma percepção mais acurada que as mulheres possuem de sua saúde, portanto elas não só buscam mais as opiniões médicas, como seguem os tratamentos propostos (SILVA; OLIVEIRA; PIERIN, 2016).

$\mathrm{Na}$ pesquisa realizada por Kern et al. (2017) foi realizada associação entre a pressão arterial dos adolescentes com os fatores de risco às doenças cardiovasculares de seus pais, que constatou prevalência de pressão arterial alterada superior entre os adolescentes cujas mães apresentavam HAS e histórico de infarto. Já entre os pais, observou-se que apenas presença de colesterol elevado associou-se com a alteração da pressão nos adolescentes.

Outra metanálise, que incluiu artigos de variados continentes, também apontou maior prevalência no sexo masculino, a qual foi associada à adiposidade abdominal e à fatores hormonais, como os níveis de testosterona (MORAES et al., 2014). Já em estudo realizado na Grécia, com amostra na faixa etária entre 9 e 13 anos, as meninas apresentaram maior susceptibilidade (MANIOS et al., 2019), divergindo dos resultados da presente pesquisa. As diferenças continentais e os hábitos de vida em populações distintas podem justificar essa divergência. 
$\mathrm{Na}$ tabela 2 observa-se que não houve associação estatisticamente significativa entre sexo e a idade nas crianças e adolescentes portadoras de hipertensão, conforme variáveis relacionadas ao estilo de vida. Ainda assim, alguns resultados podem ser destacados. Entre as pessoas que fazem uso de tabaco, verificou-se que existem menos crianças e adolescentes do sexo feminino, com idade entre 15 e 19 anos, usuárias, quando comparado às crianças e adolescentes do sexo masculino.

Tabela 2 - Associação entre sexo e a faixa etária de crianças e adolescentes com HAS, conforme estilo de vida, no período de abril de 2011 a abril de 2013, Paraíba, Brasil.

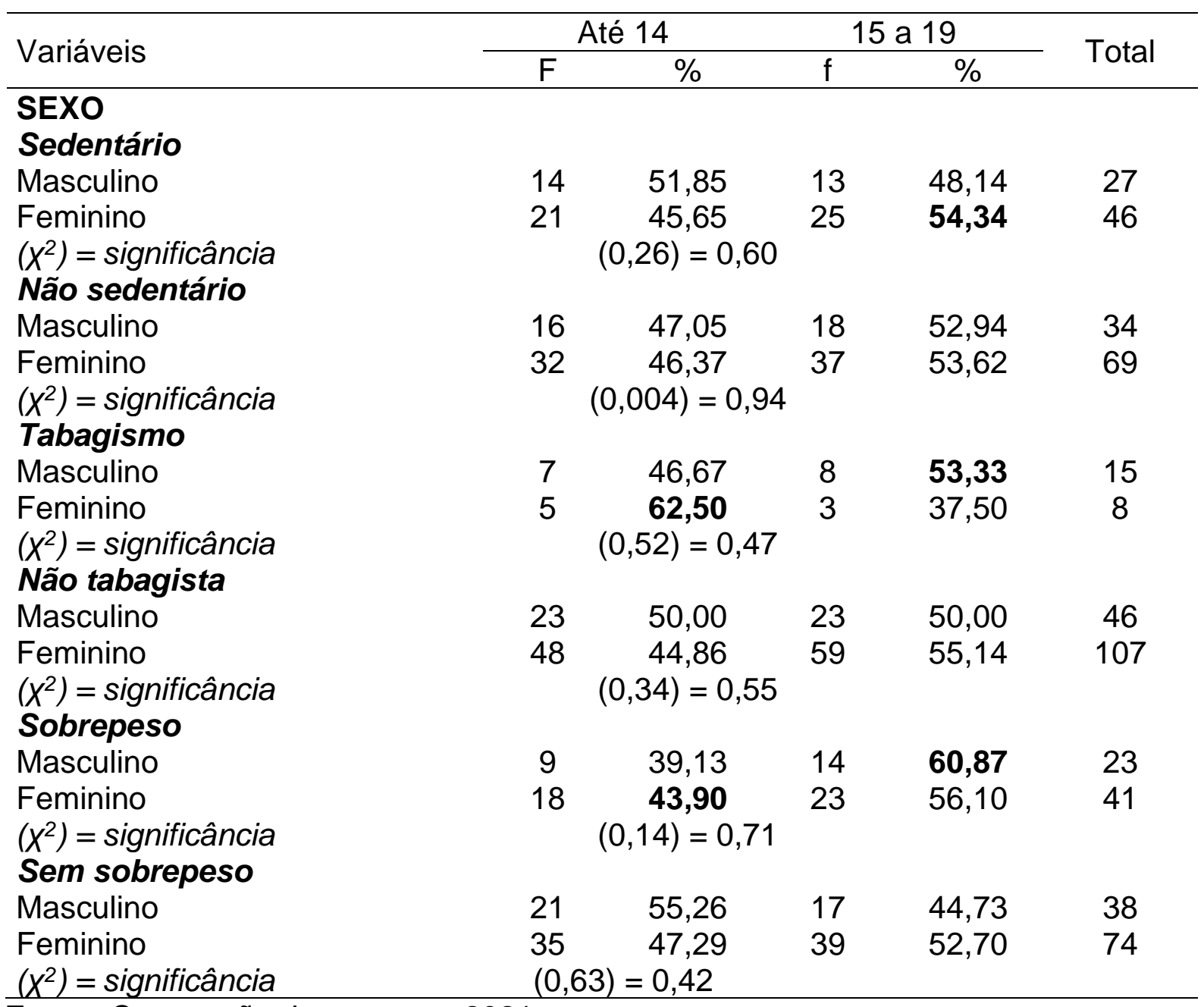

Fonte: Construção dos autores, 2021. 
Referente aos valores encontrados de acordo com o estilo de vida, que não revelaram associação estatisticamente significativa, é importante mencionar que existem outros fatores que influenciam no desenvolvimento da HAS em adolescentes. Rosas-Peralta et al. (2016) listaram as principais causas de HAS, por ordem de prevalência, em crianças e adolescentes por grupo etário, e verificaram que entre os seis e 12 anos destacaram-se doenças renais, hipertensão primária, doença vascular, causas endócrinas, coarctação da aorta e iatrogenia. No grupo de adolescentes com idade entre 12 e 18 anos as principais causas foram hipertensão primária, iatrogenia, doença renal, doença vascular renal e coarctação da aorta.

Apesar de não ser observada associação estatisticamente significativa nessa pesquisa, é notório que o sedentarismo, o tabagismo e o sobrepeso propiciam prejuízos à nossa saúde. $O$ tabagismo, assim como o etilismo, traz malefícios para o controle da pressão arterial, sendo assim, é de fácil observação que a prevalência de um descontrole pressórico é mais comum nos homens, ao notar que esses consomem mais desses produtos do que as mulheres (SILVA; OLIVEIRA; PIERIN, 2016).

No estudo de Costa et al. (2017) foi observado que ser do sexo masculino favorece a realização de atividade física com tempo igual ou superior a 150 minutos semanais. Os autores não observaram diferenças estatisticamente significativas entre o sexo e sedentarismo ou tabagismo entre os adolescentes estudados.

Em um estudo de Oliveira et al. (2019), com adolescentes entre 14 e 17 anos, a prevalência dos tabagistas foi de $5 \%$, enquanto a dos que consomem álcool ficou entre $25 \%$, mostrando um problema explícito nos hábitos dos mais jovens, que mais tarde ocasionará em vários problemas de saúde, como a HAS (OLIVEIRA et al. 2019).

Ainda em relação ao tabagismo, segundo a Organização Mundial de Saúde (2014), há prevalência no sexo masculino na faixa etária igual ou superior a 15 anos. No Brasil, dados da Pesquisa Nacional de Saúde do Escolar, com estudantes de 13 a 17 anos, revelaram a experiência precoce do brasileiro com cigarros, demonstrando que esse hábito pode ter início na adolescência. Os seus resultados também indicaram que os meninos foram maioria entre aqueles que experimentaram cigarro (IBGE, 2016). 
No presente trabalho, no grupo etário de 15 a 19 anos, considerando adolescentes hipertensos e hipertensos e diabéticos, houve concordância com esses achados, porém, deve-se destacar o uso considerável de tabaco por meninas. Em estudo recente com alunos de ensino médio de uma cidade nordestina houve predominância do sexo feminino entre fumantes, os autores sugerem gradativa equiparação entre os sexos (DANTAS et al., 2017).

Referente a outros fatores de risco, Iraheta e Bogantes (2020) explicam que o sobrepeso e o sedentarismo em crianças e adolescentes se devem aos fatos ambientais, como o exagero no uso da tecnologia em idade precoce, e que os principais colaboradores responsáveis por esses dados é um modo de vida sedentário e o alto consumo de alimentos.

O sedentarismo é justificado pela falta de exercício físico, e infelizmente nas últimas duas décadas ocorreu uma diminuição constante da atividade física na infância e na adolescência. As recomendações de saúde sugerem que os jovens pratiquem, pelo menos, sessenta minutos de exercício físico de intensidade moderada todos os dias (IRAHETA; BOGANTES, 2020). Entretanto, os resultados apresentados nessa pesquisa corroboram com a existência desses jovens que não cumprem essa recomendação, de modo que apresentam maior risco de desenvolver sobrepeso ou obesidade, e muitas outras doenças como DM e DCV.

Estudos envolvendo crianças e adolescentes indicam que distúrbios na pressão arterial e outros indicadores morfológicos de risco, como a distribuição da gordura corporal, têm potencial de emergir na adolescência. A rotina de exercícios e os hábitos que se formam durante a adolescência podem melhorar ou prejudicar seu estilo de vida e saúde na idade adulta (SCHERR et al., 2018).

No estudo de Mendes et al. (2006) foi observada associação entre presença de sedentarismo no pai ou na mãe, com a falta de atividade física no filho adolescente. Entretanto, os autores não verificaram associação entre a frequência de HAS nos pais, nas mães e nos filhos adolescentes.

Os achados da pesquisa de Scherr et al. (2018) indicam que aproximadamente $23 \%$ das crianças, com idade entre 6 e 12 anos, e $21 \%$ entre 12 e 17 anos são sobrepesas, sendo que esse aumento na prevalência de peso está ligado à fatores socioculturais e ambientais. Além disso, no grupo de adolescents, 
entre 10 e 17 anos, o presente estudo afirma existir uma influência de indivíduos com sobrepeso no aumento da pressão arterial.

Assim, como os dados apresentados na tabela 01, na tabela 03, proporcionalmente, observa-se mais crianças e adolescentes do sexo masculino hipertensos e diabéticos mais novos, do que crianças e adolescentes do sexo feminino. No entanto, sem resultado estatisticamente significativo.

Tabela 3 - Associação entre sexo e faixa etária de crianças e adolescentes com HAS e DM no período de abril de 2011 a abril de 2013, Paraíba, Brasil.

\begin{tabular}{|c|c|c|c|c|c|}
\hline \multirow{2}{*}{ Sexo } & \multicolumn{2}{|c|}{ Até 14} & \multicolumn{2}{|c|}{15 a 19} & \multirow[t]{2}{*}{ Tota } \\
\hline & $\mathrm{F}$ & $\%$ & $\mathrm{~F}$ & $\%$ & \\
\hline Masculino & 16 & 59,26 & 11 & 40,74 & 27 \\
\hline Feminino & 15 & 41,67 & 21 & 58,33 & 36 \\
\hline$\left(X^{2}\right)=$ significância & & $(1,91)=0$ & & & \\
\hline
\end{tabular}

Fonte: Construção dos autores, 2021.

Um estudo de Souza et al. (2004) evidenciou que a prevalência de diabéticos e insulino resistentes entre meninos com idade variando de 10 a 16 anos é mais baixa do que a das meninas, o que contraria a maior parte dos estudos, contudo na faixa etária entre 5 e 10 anos, notou-se que os meninos apresentam maior prevalência. Isso ocorreu pelo fato de que uma separação por idade não indica o desenvolvimento corporal do adolescente, sendo necessários meios mais amplos de análise.

Verifica-se que entre crianças e adolescentes com HAS e DM há mais sedentários e pessoas com sobrepeso no grupo das crianças e adolescentes do sexo feminino que estão entre 15 e 19 anos, se comparado às crianças e adolescentes do sexo masculino. De outra forma, mais crianças e adolescentes do sexo masculino, mais velhos, são observados entre os tabagistas. Não foram observados dados estatisticamente significativos (Tabela 4). 
Tabela 4 - Associação entre sexo e faixa etária de crianças e adolescentes com HAS e DM, conforme estilos de vida, no período de abril de 2011 a abril de 2013, Paraíba, Brasil.

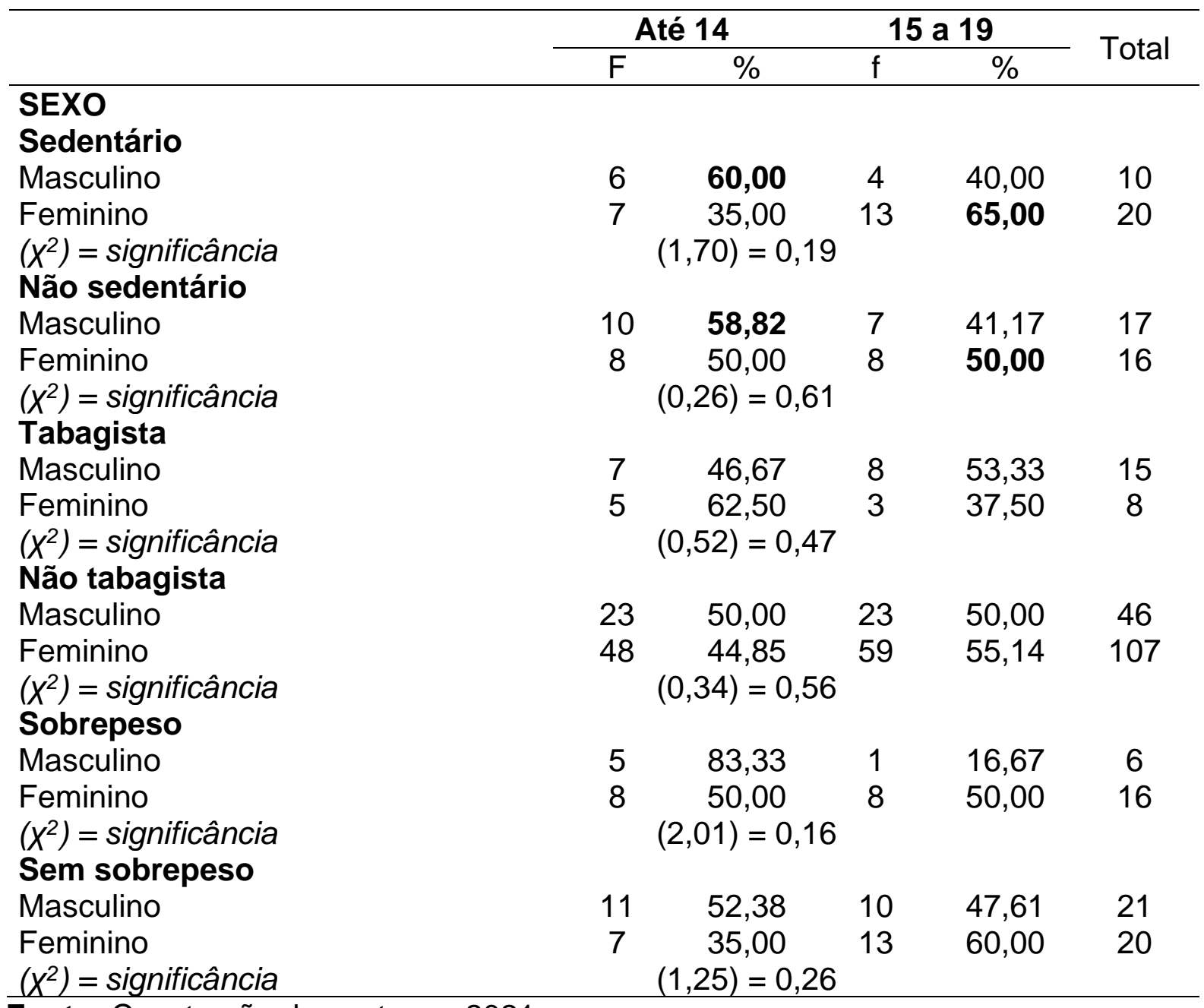

Fonte: Construção dos autores, 2021.

Entre 2005 e 2007 foram analisados casos de HAS, associados ou não ao DM, e notou-se que $0,5 \%$ dos casos estavam presentes em adolescentes na faixa etária de 14 a 19 anos de idade. A HAS na sua forma secundária pode ter início na infância, mesmo não sendo associada a esse período, ademais a sua forma primária está mais clara na adolescência e na vida adulta, assim, os hábitos adquiridos na infância e adolescência trazem fatores de risco para HAS e DM no futuro. Em decorrência, é fundamental o rastreio de crianças e adolescentes que podem 
apresentar risco aumentado, para que sejam instituídas medidas precoces para prevenção dessas doenças (GOMES; ALVES, 2009).

No estudo analisado por Costa et al. (2012) consolida que são vários os fatores envolvidos no desenvolvimento da HAS durante toda a vida, dentre eles a obesidade, o sedentarismo e os hábitos alimentares inadequados, que são os principais os principais elementos motivadores para o desenvolvimento de risco cardiovasculares que afeta o organismo a desenvolver essa doença.

Dentre os fatores evidenciados, no estudo realizado por Cardoso et al. (2015) evidencia que o estilo de vida adotado no ambiente familiar é um das principais causas para o desenvolvimento e manutenção dos comportamentos alimentares saudáveis nessa faixa etária, tendo em vista que o padrão alimentar praticado no ambiente familiar influencia na alimentação do adolescente frente a sua saúde, sendo então um forte aliado a desenvolver jovens sedentários e obesos.

\section{CONCLUSÃO}

Para as associações realizadas as variáveis relacionadas ao estilo de vida das crianças e adolescentes, considerando presença de sobrepeso, tabagismo e sedentarismo, bem como presença de HAS e/ou DM, não foram observados resultados estatisticamente significativos, mas podemos fazer algumas inferências.

A prevalência de HAS e da HAS, associada ao DM, foi maior entre o grupo das crianças e adolescentes do sexo masculino, as adolescentes com idade entre 15 e 19 anos são mais sedentárias, as crianças e adolescentes com idade até 14 anos consomem mais o tabaco, e o sobrepeso apresentou uma maior prevalência entre os adolescentes do sexo masculino no grupo com idade entre 15 e 19 anos.

A falta de informações mais atuais no DATASUS dificulta a percepção do panorama no estado da Paraíba nos últimos anos em relação às doenças cardiovasculares nos adolescentes, constituindo fator impeditivo para elaboração, implantação e implementação de políticas públicas extensivas para a promoção da saúde e prevenção de agravos nessa população estudada. 


\section{REFERÊNCIAS BIBLIOGRÁFICAS}

BITTAR, O.J. et al. Sistemas de informação em saúde e sua complexidade. Revista de Administração em Saúde, v. 18, n. 70, p.1-18, 2018.

BRASIL. Ministério da Saúde. Agência Nacional de Saúde Suplementar. Manual técnico para promoção da saúde e prevenção de riscos e doenças na saúde suplementar. Rio de Janeiro: ANS, 2011. Disponível em: http://www.ans.gov.br/images/stories/Materiais_para_pesquisa/Materiais_por_assunto/manual_p romoprev_web.pdf. Acesso em: 22 Mai 2021.

BRITO, B. B. et al. Doenças cardiovasculares: fatores de risco em adolescentes. Cogitare Enfermagem, v.21, n.2, p.1-8, 2016.

CARDOSO, S.; SANTOS, O.; NUNES, C.; LOUREIRO, I. Escolhas e hábitos alimentares em adolescentes: associação com padrões alimentares do agregado familiar. Revista Portuguesa de Saúde pública. 2015; 3(2):128-136.

COSTA, I.F.A.F. et al. Adolescentes: comportamento e risco cardiovascular. Jornal Vascular Brasileiro, v. 16, n. 3, p. 205-213, 2017.

COSTA JV, SILVA ARV, MOURA IH, CARVALHO RMN, BERNARDES LE, ALMEIDA PC. Análise de fatores de risco para hipertensão arterial em adolescentes escolares. Rev. LatinoAm. Enfermagem. 2012; 20(2): 1-7.

DANTAS, D.R.G. et al. Prevalência e Risco de Tabagismo entre Estudantes do Ensino Médio em Cidade do Nordeste do Brasil. Portuguese Journal of Public Health, v. 35, n. 1, p.44-51, 2017.

FARIAS JÚNIOR, J.C.et al. Fatores de risco cardiovascular em adolescentes: prevalência e associação com fatores sociodemográficos. Revista Brasileira de Epidemiologia, v. 14, n. 1, p. $50-62,2011$.

FONTES, V.S.; NEVES, F.S.; CÂNDIDO, A.P.C. Quemerina e fatores relacionados ao risco cardiovascular em crianças e adolescentes: uma revisão sistemática. Revista Paulista de Pediatria, v.36, n.2, p.221 -229, 2018.

FRONTEIRAS, I. Estudos observacionais na era de medicina baseada em evidências: Breve revisão sobre a relevância, taxonomia e desenhos. Revista científica de ordens dos médicos, Portugal, v.26, n.1, p.161-170, 2013.

GOMES, B.M.R.; ALVES, J.G.B. Prevalência de hipertensão arterial e fatores associados em estudantes de Ensino Médio de escolas públicas da Região Metropolitana do Recife, Pernambuco, Brasil, 2006. Cadernos de Saúde Pública, v. 25, p. 375-381, 2009.

GONÇALVES, V.S.S. et al. Prevalência de hipertensão arterial entre adolescentes: revisão sistemática e metanálise. Revista de Saúde Pública, v. 50, n. 27, p. 1-12, 2016. DOI:10.1590/S1518-8787.2016050006236. Disponível em: https://www.scielo.br/j/rsp/a/PMXByt66YcRmL7NzBbfkLwC/?format=pdf\&lang=pt . Acesso em: 10 jan. 2021.

INSTITUTO BRASILEIRO DE GEOGRAFIA E ESTATÍSTICA (IBGE). Pesquisa Nacional de Saúde do Escolar (PeNSE) 2015. Rio de Janeiro: IBGE, 2016. 126 p. Disponível em: https://www.ibge.gov.br/estatisticas/sociais/populacao/9134-pesquisa-nacional-de-saude-doescolar.html?=\&t=0-que-e Acesso em: 19 mar 2021. 
IRAHETA, B.E.; BOGANTES, C.A. Análisis del sobrepeso y obesidad, niveles de actividad física y autoestima de La niñez salvadoreña. MHSalud, v.17, n.1, p.1-18, 2020.

KERN, D.G. et al. Pressão arterial alterada em adolescentes: associação com fatores de risco às doenças cardiovasculares de seus pais. Revista Saúde e Pesquisa, v. 10, n. 1, p. 157-162, 2017.

MANIOS, Y. et al. Lifestyle, anthropometric, socio-demographic and perinatal correlates of early adolescence hypertension: The Healthy Growth Study. Nutrition, Metabolism \& Cardiovascular Diseases, v. 29, n. 2, p. 159-169, 2019.

MARTE, A.P.; SANTOS, R.D. Bases fisiopatológicas da dislipidemia e hipertensão arterial. Revista Brasileira de Hipertensão, v. 14, n. 4, p. 252-7, 2007.

MENDES, M.J.F.L. et al. Associação de fatores de risco para doenças cardiovasculares em adolescentes e seus pais. Revista Brasileira de Saúde Materno Infantil, v.9, p. S49-S54, 2006. Supl. 1.

MORAES, A.C.F. et al. Prevalence of High Blood Pressure in 122,053 Adolescents: A Systematic Review and Meta-Regression. Medicine, v. 93, n. 27, p. 1-10, 2014.

OLIVEIRA, G. et al. Agregação dos fatores de risco cardiovascular: álcool, fumo, excesso de peso e sono de curta duração em adolescentes do estudo ERICA. Caderno Saúde Pública, v. 35, n. 12, p. 2 - 12, 2019.

ROSAS-PERALTA, M. et al. Systemic arterial hypertension in child and adolescent. Revista Médica del Instituto Mexicano del Seguro Social, v. 54 suppl1: S52-66, p. 1 - 15, 2016.

SCHERR, C. Programa do Ginásio Experimental Olímpico e sua Associação com a Prevalência de Fatores de Risco Cardiovascular em Adolescentes: Estudo Transversal. Arquivos Brasileiros de Cardiologia, v.112, n.6, p.775 - 781, 2019.

SCHERR, C. et al. Práticas Esportivas e Risco Cardiovascular em Adolescentes. Arquivos Brasileiros de Cardiologia, v. 110, n. 3, p. 248-255, 2018.

SENNA, S.R.C.M.; DESSEN, M.A. Reflexões sobre a saúde do adolescente brasileiro. Psicologia, Saúde \& Doenças, v. 16, n. 2, p. 217 - 229, 2015.

SILVA, P.M.S.; CIRINO NETO, J.; MIRANDA, G.M.D. Perfil epidemiológico de sífilis congênita em um Estado do Nordeste. Revista Fontes Documentais, v. 3, p. 512-519, 2020.

SILVA, P.M.S.; AUTRAN, M.M.M. Repositório Datasus: organização e relevância dos dados abertos em saúde para a vigilância epidemiológica. P2P E INOVAÇÃO, v. 6, p. 50-59, 2019.

SILVA, S.S.B. E.; OLIVEIRA, S.F.S.B.; PIERIN, A.M.G. O controle da hipertensão em homens e mulheres: uma análise comparativa. Revista Escola de Enfermagem USP, v. 50, n. 1, p. 50-58, 2016.

SOUZA, M.R. et al. Análise da prevalência de resistência insulínica e diabetes mellitus tipo $2 \mathrm{em}$ crianças e adolescentes obesos. Arquivos de Ciências da Saúde, v. 11, n.4, p. 215-8, 2004.

\section{Agradecimentos}

Conselho Nacional de Desenvolvimento Científico e Tecnológico (CNPq) por meio do Programa Institucional de Bolsas de Iniciação Científica PIBIC/CNPq-UFCG. 